\title{
A characterization of rational numbers by $p$-adic Sylvester series expansions
}

\author{
by \\ ViCHIAN LAOHAKOSOL (Bangkok) and \\ NARAKORn Rompurk Kanasri (Khon Kaen)
}

1. Introduction. In [2] and [3], A. Knopfmacher and J. Knopfmacher introduce a general algorithm which leads to series expansions of $p$-adic numbers as sums of rational numbers. The problem of characterizing rational numbers by such expansions is of interest here. The Knopfmachers in [3] are able to establish a proposition which implies that, subject to a restriction on the so-called digit set, two particular classes of their series expansions, namely, the $p$-adic Sylvester and the $p$-adic Engel expansions are finite if and only if they represent rational numbers. Subsequently in [1], a complete characterization of rational numbers, without the restriction on the digit set, via their $p$-adic Engel series expansions is proved, while the one via $p$-adic Sylvester series expansions is still open. In another direction, a complete characterization of rational numbers via their $p$-adic Lüroth series expansion was established in [4]. The objective of this article is to complement these earlier works and fill a gap by establishing a complete characterization of rational numbers via their $p$-adic Sylvester series expansions. We mention in passing that the situation in function fields, i.e., fields of formal Laurent series over a finite filed, is simpler: it is shown in [5] that an element in a function field is rational if and only if its Sylvester series expansion is finite.

Let us recall Knopfmachers' algorithm for constructing series expansion in the $p$-adic number field. Let $p$ be a fixed prime, $\mathbb{Q}_{p}$ the field of $p$-adic numbers equipped with the $p$-adic valuation $|\cdot|_{p}$, normalized so that $|p|_{p}=$ $p^{-1}$, and let $\mathbb{Z}_{p}$ be the ring of $p$-adic integers. For $A \in \mathbb{Q}_{p}$, define the order $v_{p}(A)$ of $A$ by $|A|_{p}=p^{-v_{p}(A)}$, and $v_{p}(0)=+\infty$. Each $A \in \mathbb{Q}_{p} \backslash\{0\}$ is uniquely representable in the form

2000 Mathematics Subject Classification: 11A67, 11J61.

Key words and phrases: p-adic number, Sylvester series expansion, Knopfmachers' algorithm. 


$$
A=\sum_{n=v_{p}(A)}^{\infty} c_{n} p^{n}, \quad c_{n} \in\{0,1, \ldots, p-1\}, c_{v_{p}(A)} \neq 0 .
$$

The fractional part, $\langle A\rangle$, of $A$ is defined as the finite series

$$
\langle A\rangle= \begin{cases}\sum_{n=v_{p}(A)}^{0} c_{n} p^{n} & \text { if } v_{p}(A) \leq 0, \\ 0 & \text { if } v_{p}(A)>0 .\end{cases}
$$

Denote the set of all fractional parts by

$$
S_{p}:=\left\{\langle A\rangle ; A \in \mathbb{Q}_{p}\right\} \subset \mathbb{Q} .
$$

The Knopfmachers' series expansion algorithm proceeds as follows: for $A$ in $\mathbb{Q}_{p}$, let $a_{0}:=\langle A\rangle \in S_{p}$. Define $A_{1}:=A-a_{0}$. If $A_{n} \neq 0(n \geq 1)$ is already defined, put

$$
a_{n}=\left\langle\frac{1}{A_{n}}\right\rangle \in S_{p}
$$

and

$$
A_{n+1}=\left(A_{n}-\frac{1}{a_{n}}\right) \frac{s_{n}}{r_{n}}
$$

if $a_{n} \neq 0$, where $r_{n}, s_{n}$ are non-zero rational numbers which may depend on $a_{1}, \ldots, a_{n}$. Then

$$
\begin{aligned}
A & =a_{0}+A_{1}=a_{0}+\frac{1}{a_{1}}+\frac{r_{1}}{s_{1}} A_{2}=\cdots \\
& =a_{0}+\frac{1}{a_{1}}+\frac{r_{1}}{s_{1}} \frac{1}{a_{2}}+\cdots+\frac{r_{1} \cdots r_{n-1}}{s_{1} \cdots s_{n-1}} \frac{1}{a_{n}}+\frac{r_{1} \cdots r_{n}}{s_{1} \cdots s_{n}} A_{n+1} .
\end{aligned}
$$

The process ends in a finite expansion if some $A_{n+1}=0$. If some $a_{n}=0$, then $A_{n+1}$ is not defined. This is ruled out by imposing the condition

$$
v_{p}\left(s_{n}\right)-v_{p}\left(r_{n}\right) \geq 2 v_{p}\left(a_{n}\right)-1
$$

Thus

$$
A=a_{0}+\frac{1}{a_{1}}+\sum_{n=1}^{\infty} \frac{r_{1} \cdots r_{n}}{s_{1} \cdots s_{n}} \frac{1}{a_{n+1}} .
$$

We are interested here in the case where $r_{n}=s_{n}=1$. In this case, the algorithm yields a well-defined (with respect to the $p$-adic valuation) and unique series expansion, termed $p$-adic Sylvester series expansion or $S$-series for short, of the form

$$
A=a_{0}+\sum_{n=1}^{\infty} \frac{1}{a_{n}}
$$

where the digits $a_{n}$ are, by the algorithm, subject to the restrictions

$$
a_{0}=\langle A\rangle
$$




$$
\begin{aligned}
a_{n}=\frac{c_{\nu_{n}}}{p^{\nu_{n}}} & +\cdots+\frac{c_{1}}{p}+c_{0} \in S_{p} \\
\nu_{n} & :=-v_{p}\left(a_{n}\right), \quad c_{i} \in\{0,1, \ldots, p-1\}, \quad c_{\nu_{n}} \neq 0 \quad(n \geq 1) .
\end{aligned}
$$

For $A_{n} \neq 0$, writing $1 / A_{n}=a_{n}+\sum_{r \geq 1} c_{r}^{\prime} p^{r}$, say, we get

$$
v_{p}\left(a_{n} A_{n}-1\right)=v_{p}\left(A_{n} \sum_{r \geq 1} c_{r}^{\prime} p^{r}\right) \geq v_{p}\left(A_{n}\right)+1
$$

and, with $\nu_{n}=-v_{p}\left(a_{n}\right) \in \mathbb{N}$, it is easily checked by induction that

$$
\nu_{n} \geq 2^{n}-1 \quad \text { and } \quad \nu_{n+1} \geq 2 \nu_{n}+1 \quad(n \geq 1)
$$

This last inequality shows that the digits $a_{n}$ are of strictly decreasing order and so an infinite $\mathrm{S}$-series can never be periodic. For $r \in \mathbb{N}$, define

$$
\begin{aligned}
D_{r}:=\left\{b_{r}:=c_{r}+c_{r-1} p+\cdots+c_{0} p^{r}\right. & \\
c_{i} & \left.\in\{0,1, \ldots, p-1\}(i=0,1, \ldots, r), c_{r} \neq 0\right\} .
\end{aligned}
$$

Note that each $b_{r} \in D_{r}$ is bounded below and above by

$$
1 \leq b_{r} \leq(p-1)\left(1+p+\cdots+p^{r}\right)=p^{r+1}-1<p^{r+1} .
$$

To derive a characterization of rational numbers via their S-series, it clearly suffices to consider only those numbers belonging to $p \mathbb{Z}_{p} \backslash\{0\}$. Our characterization reads:

Theorem 1. Let $A \in p \mathbb{Z}_{p} \backslash\{0\}$. Then $A \in \mathbb{Q}$ if and only if its $S$-series is either finite, or of the form

$$
A=\frac{1}{a_{1}}+\cdots+\frac{1}{a_{n}}-\frac{p^{\nu_{n+1}}}{\beta_{n+1}} \quad(n \in \mathbb{N}),
$$

where $\beta_{n+1} \in \mathbb{N}, p \nmid \beta_{n+1}, \beta_{n+1}<p^{\nu_{n+1}+1}$, and the tail of $A$ is of the form

$$
-\frac{p^{r}}{\beta}=\frac{p^{r(1)}}{b_{r(1)}}+\cdots+\frac{p^{r(i)}}{b_{r(i)}}-\frac{p^{r(i+1)}}{\beta b_{r(1)} \cdots b_{r(i)}}=\sum_{i=1}^{\infty} \frac{p^{r(i)}}{b_{r(i)}},
$$

where $\beta \in \mathbb{N}, p \nmid \beta, \beta<p^{r+1}, r(1)=r \in \mathbb{N}, r(i+1)=2 r(i)+1$,

$$
b_{r}=p^{r+1}-\beta, \quad b_{r(i+1)}=p^{r(i+1)+1}-\beta b_{r(1)} \cdots b_{r(i)} \quad(i \geq 1) .
$$

2. Proof of the theorem. That $A$ having an S-series finite or of the form as stated in the theorem implies that $A$ is rational is easily checked.

It remains to show the converse, i.e., starting with any $A=: A_{1} \in \mathbb{Q}$, we show that its $\mathrm{S}$-series expansion must either be finite or infinite with tail as stated in the theorem. Keeping the notation of the last section, note that $A_{1} \in \mathbb{Q}$ implies that $A_{n} \in \mathbb{Q}$ for all $n \geq 2$, and write

$$
A_{n}=p^{\nu_{n}} \frac{\alpha_{n}}{\beta_{n}}
$$

where $\alpha_{n} \in \mathbb{Z}, \beta_{n} \in \mathbb{N}, \operatorname{gcd}\left(\alpha_{n}, \beta_{n}\right)=1$ and $p \nmid \alpha_{n} \beta_{n}$. 
Since the remaining proof is quite involved, we proceed stepwise by establishing several lemmas and begin with a simplification.

LEmma 2. If $\alpha_{n}$ and $\alpha_{n+1}$ are both $>0$, then $\alpha_{n+1}<\alpha_{n}$. Moreover, if $\alpha_{n}<0$, then $\alpha_{n+1}<0$.

Proof. From the recurrence relation (1), we have $A_{n+1}=A_{n}-1 / a_{n}$, i.e.,

$$
p^{\nu_{n+1}-\nu_{n}} \frac{\alpha_{n+1}}{\beta_{n+1}}=\frac{\alpha_{n} b_{\nu_{n}}-\beta_{n}}{b_{\nu_{n}} \beta_{n}} .
$$

Since the left-hand fraction is in lowest terms, we get

$$
p^{\nu_{n+1}-\nu_{n}} \alpha_{n+1} \leq \alpha_{n} b_{\nu_{n}}-\beta_{n}<\alpha_{n} b_{\nu_{n}},
$$

so $\alpha_{n+1}<\alpha_{n}$, and the first assertion is proved. The second assertion follows at once from (6).

From Lemma 2 and the recurrence relation (1), if we start off with $A=$ $A_{1}=p^{\nu_{1}} \alpha_{1} / \beta_{1}>0$, then $\alpha_{1}>0$, and since $A$ is a rational integer, eventually for some $m \in \mathbb{N}$ we must have either $\alpha_{m}=0$, which yields a terminating expansion, or $\alpha_{m}<0$, which yields $\alpha_{k}<0$ for all $k \geq m$, resulting in an infinite expansion.

Henceforth, without loss of generality, we assume that all the numerators are negative, i.e., $\alpha_{n}<0(n \geq 1)$.

Our next lemma shows that certain negative rational numbers have infinite S-series of a specific form which will be typical for what follows.

Lemma 3. Let $r, \beta \in \mathbb{N}$ with $p \nmid \beta$ and $\beta<p^{r+1}$. Then we have the following unique $S$-series representation:

$$
-\frac{p^{r}}{\beta}=\frac{p^{r(1)}}{b_{r(1)}}+\cdots+\frac{p^{r(i)}}{b_{r(i)}}-\frac{p^{r(i+1)}}{\beta b_{r(1)} \cdots b_{r(i)}}=\sum_{i=1}^{\infty} \frac{p^{r(i)}}{b_{r(i)}},
$$

where $r(1)=r, r(i+1)=2 r(i)+1, b_{r}=p^{r+1}-\beta$, and

$$
b_{r(i+1)}=p^{r(i+1)+1}-\beta b_{r(1)} \cdots b_{r(i)} \quad(i \geq 1) .
$$

Proof. Observe first that all $b_{r}$ are in $D_{r}$. The result follows easily from the following chain of relations:

$$
\begin{aligned}
-\frac{p^{r}}{\beta} & =\frac{p^{r(1)}}{b_{r(1)}}-p^{r(1)}\left(\frac{1}{\beta}+\frac{1}{b_{r(1)}}\right)=\frac{p^{r(1)}}{b_{r(1)}}-\frac{p^{r(2)}}{\beta b_{r(1)}} \\
& =\frac{p^{r(1)}}{b_{r(1)}}+\frac{p^{r(2)}}{b_{r(2)}}-p^{r(2)}\left(\frac{1}{\beta b_{r(1)}}+\frac{1}{b_{r(2)}}\right)=\frac{p^{r(1)}}{b_{r(1)}}+\frac{p^{r(2)}}{b_{r(2)}}-\frac{p^{r(3)}}{\beta b_{r(1)} b_{r(2)}} \\
& =\cdots,
\end{aligned}
$$

upon noting that $\beta b_{r(1)} \cdots b_{r(i)}<p^{r(i+1)+1}$. The uniqueness is immediate by construction. 
The next lemma yields a complete characterization for the case where the denominator, $\beta$, belongs to our starting range.

Lemma 4. If $A=-p^{\nu} \alpha / \beta<0 ; \nu, \alpha, \beta \in \mathbb{N} ; p \nmid \alpha \beta$; $\operatorname{gcd}(\alpha, \beta)=1$ and $\beta<p^{\nu+1}$, then $A$ has an $S$-series expansion of the form

$$
A=\frac{1}{a_{1}}+\cdots+\frac{1}{a_{n}}-\frac{p^{\nu_{n+1}}}{\beta_{n+1}} \quad(n \in \mathbb{N}),
$$

where $\beta_{n+1} \in \mathbb{N}, p \nmid \beta_{n+1}, \beta_{n+1}<p^{\nu_{n+1}+1}$, and so the tail of $A$ is as specified in Lemma 3.

Proof. Let $-p^{\nu} \alpha / \beta=A=: A_{1}=p^{\nu_{1}} \alpha_{1} / \beta_{1}<0$. The recurrence relation (1) is $A_{n+1}=A_{n}-1 / a_{n}$, implying that $A_{n}<0$ for all $n \geq 2$ and so the S-series of $A$ must be infinite. Observe that for each $a_{n} \in S_{p}$, we have

$$
0<a_{n}<\sum_{k=0}^{\infty} \frac{p-1}{p^{k}}=p,
$$

and so we can write $a_{n}=b_{\nu_{n}} / p^{\nu_{n}}$, where $b_{\nu_{n}} \in D_{\nu_{n}}$. Thus,

$$
0<b_{\nu_{n}} \leq p^{\nu_{n}+1}-1<p^{\nu_{n}+1} \text {. }
$$

Substituting $A_{n}=p^{\nu_{n}} \alpha_{n} / \beta_{n}$ into the recurrence relation (1), we get

$$
b_{\nu_{n}} \alpha_{n+1} p^{\nu_{n+1}-\nu_{n}} \beta_{n}=\left(b_{\nu_{n}} \alpha_{n}-\beta_{n}\right) \beta_{n+1} .
$$

Since $\operatorname{gcd}\left(\alpha_{n+1} p^{\nu_{n+1}-\nu_{n}}, \beta_{n+1}\right)=1$, we have $\beta_{n+1} \mid b_{\nu_{n}} \beta_{n}$ and so

$$
\left|\alpha_{n+1} p^{\nu_{n+1}-\nu_{n}}\right| \leq\left|\frac{b_{\nu_{n}} \alpha_{n+1} p^{\nu_{n+1}-\nu_{n}} \beta_{n}}{\beta_{n+1}}\right|=\left|b_{\nu_{n}} \alpha_{n}-\beta_{n}\right| \leq\left|b_{\nu_{n}} \alpha_{n}\right|+\beta_{n} .
$$

Here and throughout, $|\cdot|$ denotes the usual absolute value. Since

$$
\nu_{n+1} \geq 2 \nu_{n}+1 \geq \nu_{n}+2 \nu_{n-1}+2 \geq \cdots \geq \nu_{n}+\nu_{n-1}+\cdots+2 \nu_{1}+n,
$$

we get

$$
\left|\alpha_{n+1}\right|<\left|\alpha_{n}\right|+\frac{\beta_{n}}{p^{\nu_{n+1}-\nu_{n}}} .
$$

From $\beta_{n+1} \mid b_{n} \beta_{n}$, we deduce that

$$
0<\beta_{n+1} \leq b_{\nu_{n}} \beta_{n} \leq \cdots \leq b_{\nu_{n}} b_{\nu_{n-1}} \cdots b_{\nu_{1}} \beta_{1}<p^{\nu_{n}+\nu_{n-1}+\cdots+\nu_{1}+n} \beta_{1} .
$$

Thus, by (8) and $\beta_{1}<p^{\nu_{1}+1}$,

$$
\frac{\beta_{n}}{p^{\nu_{n+1}-\nu_{n}}} \leq \frac{\beta_{n}}{p^{\nu_{n}+1}}<\frac{p^{\nu_{n-1}+\cdots+\nu_{1}+n-1} \beta_{1}}{p^{\nu_{n}+1}}<1,
$$

and so

$$
\left|\alpha_{n+1}\right| \leq\left|\alpha_{n}\right|
$$

It follows that for all $n$ sufficiently large, $-\alpha_{n}=\alpha \in \mathbb{N}$ and the relation (7) becomes

$$
-b_{\nu_{n}} \alpha p^{\nu_{n+1}-\nu_{n}} \beta_{n}=\left(-b_{\nu_{n}} \alpha-\beta_{n}\right) \beta_{n+1}
$$


Since $\operatorname{gcd}\left(\alpha, \beta_{n}\right)=\operatorname{gcd}\left(\alpha, \beta_{n+1}\right)=1$, this implies $\alpha=1$. Consequently, for all large $n$ we must have $\alpha_{n}=-1$, and the desired result follows.

The next lemma will ensure that the construction of S-series does not technically enlarge the denominator.

Lemma 5. Let $M, N(\geq 0), \alpha(\geq 2), \nu(\geq 1)$ be integers satisfying $0<$ $(\alpha+M) / 2(\alpha+N)<1$. Let $x, y \in D_{\nu}$, and define

$$
B(y):=(\alpha+M) p^{\nu+1} y-(\alpha+N) y^{2} \quad\left(y \in D_{\nu}\right) .
$$

Assume that

$$
x-(\alpha+N) y=-(\alpha-k) p^{\nu+1},
$$

where $k \in\{-N+1, \ldots, \alpha-1, \alpha\}$. Then

$$
\max _{y \in D_{\nu}} B(y)=\frac{(M+k)(\alpha-k)}{\alpha+N} p^{2 \nu+2}-\frac{\alpha-2 k-M}{\alpha+N} p^{\nu+1} x-\frac{x^{2}}{\alpha+N} .
$$

Proof. Since $B(y)$ represents a concave parabola, the sought-after maximum, with $y \in \mathbb{Z}$, must occur at

$$
y=\left[\frac{\alpha+M}{2(\alpha+N)} p^{\nu+1}\right]+\{0 \text { or } 1\},
$$

where $[x]$ denotes the integer part of $x \in \mathbb{R}$. Write

$$
y=\frac{\alpha+M}{2(\alpha+N)} p^{\nu+1}+\varepsilon
$$

Direct computation yields

$$
\max _{y \in D_{\nu}} B(y)=\frac{(\alpha+M)^{2}}{4(\alpha+N)} p^{2 \nu+2}-\varepsilon^{2}(\alpha+N) .
$$

Using the assumption (13), we get

$$
\varepsilon=\frac{\alpha-2 k-M}{2(\alpha+N)} p^{\nu+1}+\frac{x}{\alpha+N} .
$$

Substituting into (14) and using $x \in D_{\nu}$, we get the desired result.

The final step now is to enlarge the denominator, $\beta$, by induction on its range.

\section{LEMMA 6.}

(i) If $A=-p^{\nu} / \beta<0 ; \nu, \beta, \mu(\geq 2)$ are positive integers; $p \nmid \beta$ and $0<\beta<\mu p^{\nu+1}$, then $A$ has an infinite $S$-series expansion with tail as in Lemma 3.

(ii) If $A=-\alpha p^{\nu} / \beta<0 ; \nu, \alpha(\geq 2), \beta, \mu(\geq 2)$ are positive integers; $p \nmid \alpha \mu \beta, \operatorname{gcd}(\alpha, \beta)=1$ and $0<\beta<\mu p^{\nu+1}$, then $A$ has an infinite $S$-series expansion with tail as in Lemma 3 . 
Proof. We proceed by induction on $\mu \in \mathbb{N}$ assuming that (i)-(ii) hold for $0<\beta<(\mu-1) p^{\nu+1}$, with the starting cases, $\mu=1$, already settled in Lemma 4 . It suffices then to consider the case where $(\mu-1) p^{\nu+1}<\beta<\mu p^{\nu+1}$, $\mu \geq 2$, from which we write

$$
\beta=(\mu-1) p^{\nu+1}+x_{1} \quad \text { with } 0<x_{1}<p^{\nu+1} .
$$

(i) We note the identity

$$
\begin{aligned}
-\frac{p^{\nu}}{\beta} & =-\frac{p^{\nu}}{(\mu-1) p^{\nu+1}+x_{1}} \\
& =\frac{p^{\nu}}{p^{\nu+1}-x_{1}}-p^{\nu}\left(\frac{1}{(\mu-1) p^{\nu+1}+x_{1}}+\frac{1}{p^{\nu+1}-x_{1}}\right) \\
& =\frac{p^{\nu}}{p^{\nu+1}-x_{1}}-\frac{\mu p^{2 \nu+1}}{(\mu-1) p^{2 \nu+2}-(\mu-2) x_{1} p^{\nu+1}-x_{1}^{2}} \\
& =\frac{p^{\nu}}{p^{\nu+1}-x_{1}}+\frac{p^{\nu 2} \alpha_{2}}{\beta_{2}},
\end{aligned}
$$

where $p^{\nu_{2}} \alpha_{2} / \beta_{2}$, with $\nu_{2} \geq 2 \nu+1$, is in reduced fraction form. Since $\mu \geq 2$, we have

$$
\beta_{2} \leq(\mu-1) p^{2 \nu+2}-(\mu-2) x_{1} p^{\nu+1}-x_{1}^{2}<(\mu-1) p^{2 \nu+2},
$$

and invoking the induction hypothesis we finish the proof of (i).

(ii) In this part, we assume in addition that the result holds for all smaller $\alpha$-parts, which are the absolute values of the numerators, with the starting case $\alpha=1$ being confirmed in (i) and the denominator in the range in question.

Write $\nu:=\nu_{1}, \beta:=\beta_{1}$. The construction gives

$$
\begin{aligned}
& A=: A_{1}=-\frac{\alpha p^{\nu_{1}}}{\beta_{1}}=:-\frac{\alpha p^{\nu_{1}}}{(\mu-1) p^{\nu_{1}+1}+x_{1}} \\
= & \frac{p^{\nu_{1}}}{p^{\nu_{1}+1}-y_{1}}-p^{\nu_{1}}\left\{\frac{\alpha}{(\mu-1) p^{\nu_{1}+1}+x_{1}}+\frac{1}{p^{\nu_{1}+1}-y_{1}}\right\} \\
= & \frac{p^{\nu_{1}}}{p^{\nu_{1}+1}-y_{1}}-p^{\nu_{1}}\left\{\frac{(\alpha+\mu-1) p^{\nu_{1}+1}+x_{1}-\alpha y_{1}}{(\mu-1) p^{2\left(\nu_{1}+1\right)}+\left\{x_{1}-(\mu-1) y_{1}\right\} p^{\nu_{1}+1}-x_{1} y_{1}}\right\} \\
= & : \frac{p^{\nu_{1}}}{p^{\nu_{1}+1}-y_{1}}+A_{2} .
\end{aligned}
$$

By the algorithm, $p^{\nu_{1}+1} \mid\left(x_{1}-\alpha y_{1}\right)$, and since $x_{1}, y_{1} \in D_{\nu_{1}}$, we must have

$$
x_{1}-\alpha y_{1} \in\left\{-(\alpha-1) p^{\nu_{1}+1},-(\alpha-2) p^{\nu_{1}+1}, \ldots,-p^{\nu_{1}+1}, 0\right\} .
$$

Substituting

$$
x_{1}-\alpha y_{1}=-\left(\alpha-k_{1}\right) p^{\nu_{1}+1}, \quad k_{1} \in\{1, \ldots, \alpha\},
$$


into (15), we get

$$
\begin{aligned}
A_{2} & =\frac{-\left(k_{1}+\mu-1\right) p^{2 \nu_{1}+1}}{\left(\mu+k_{1}-\alpha-1\right) p^{2 \nu_{1}+2}+\left(2 \alpha-\mu-k_{1}+1\right) p^{\nu_{1}+1} y_{1}-\alpha y_{1}^{2}} \\
& =: \frac{\alpha_{2} p^{\nu_{2}}}{\beta_{2}} .
\end{aligned}
$$

At this point, we pause to make some important observations.

(a) If $2 \alpha-\mu-k_{1}+1 \leq 0$, then

$\beta_{2} \leq\left(\mu+k_{1}-\alpha-1\right) p^{2 \nu_{1}+2}+\left(2 \alpha-\mu-k_{1}+1\right) p^{\nu_{1}+1} y_{1}-\alpha y_{1}^{2}<(\mu-1) p^{2 \nu_{1}+2}$, i.e., $\beta_{2}$ falls inside the previous range, and we are done by induction. We thus assume that $2 \alpha-\mu-k_{1}+1>0$. By Lemma 5 , we have

$$
\begin{aligned}
\beta_{2} & <\left\{\mu+k_{1}-\alpha-1+\frac{(\alpha-\mu+1)\left(\alpha-k_{1}\right)}{\alpha}\right\} p^{2 \nu_{1}+2}-\frac{\mu-k_{1}-1}{\alpha} p^{\nu_{1}+1} x_{1} \\
& =\frac{k_{1}(\mu-1)}{\alpha} p^{2 \nu_{1}+2}-\frac{\mu-1-k_{1}}{\alpha} p^{\nu_{1}+1} x_{1} .
\end{aligned}
$$

If $k_{1} \leq \mu-1$, then

$$
\beta_{2}<\frac{k_{1}(\mu-1)}{\alpha} p^{2 \nu_{1}+2} \leq(\mu-1) p^{2 \nu_{1}+2} .
$$

Thus, $\beta_{2}$ falls inside the previous inductive range and the induction hypothesis ends this case. If $k_{1} \geq \mu$, then

$$
\beta_{2}<\frac{k_{1}(\mu-1)}{\alpha} p^{2 \nu_{1}+2}+\frac{k_{1}-\mu+1}{\alpha} p^{2 \nu_{1}+2}=\frac{k_{1} \mu-\mu+1}{\alpha} p^{2 \nu_{1}+2},
$$

which is $\leq(\mu-1) p^{2 \nu_{1}+2}$ if and only if $k_{1} \leq(\alpha+1)(\mu-1) / \mu$. These considerations show that, without loss of generality, we may assume $\alpha \geq \mu$.

(b) If $p \mid\left(k_{1}+\mu-1\right)$, then the power of the prime $p$ in the numerator of (16) is larger, i.e., $\nu_{2} \geq 2 \nu_{1}+2$, which forces $\beta_{2}$ to fall inside the previous range because, by (17),

$$
\beta_{2}<\frac{k_{1} \mu-\mu+1}{\alpha} p^{2 \nu_{1}+2} \leq(\mu-1) p^{2 \nu_{1}+2},
$$

and we are done by induction.

(c) If the numerator and denominator in (16) are not relatively prime, i.e.,

$\operatorname{gcd}\left(k_{1}+\mu-1,\left(\mu+k_{1}-\alpha-1\right) p^{2 \nu_{1}+2}+\left(2 \alpha-\mu-k_{1}+1\right) p^{\nu_{1}+1} y_{1}-\alpha y_{1}^{2}\right)>1$ then there is a cancellation between the numerator and the denominator in (16) and, using also (17), the new denominator is less than

$$
\frac{k_{1} \mu-\mu+1}{2 \alpha} p^{2 \nu_{1}+2} \leq(\mu-1) p^{2 \nu_{1}+2},
$$

and we are done again by induction. 
(d) The range of the new denominator $\beta_{2}$ is not technically enlarged because for $\mu \geq 2$, we always have by (17),

$$
\beta_{2}<\frac{k_{1} \mu-\mu+1}{\alpha} p^{2 \nu_{1}+2} \leq \mu p^{2 \nu_{1}+2} .
$$

(e) From observations (b) and (c), it suffices to consider only those $A_{2}$ whose corresponding $k_{1}$ satisfies both $\operatorname{gcd}\left(k_{1}+\mu-1, p\right)=1$ and $\operatorname{gcd}\left(k_{1}+\mu-1,\left(\mu+k_{1}-\alpha-1\right) p^{2 \nu_{1}+2}+\left(2 \alpha-\mu-k_{1}+1\right) p^{\nu_{1}+1} y_{1}-\alpha y_{1}^{2}\right)=1$. This being so, we must have $\nu_{2}=2 \nu_{1}+1, \alpha_{2}=-\left(k_{1}+\mu-1\right)$ and

$$
\begin{aligned}
\beta_{2} & =\left(\mu+k_{1}-\alpha-1\right) p^{2 \nu_{1}+2}+\left(2 \alpha-\mu-k_{1}+1\right) p^{\nu_{1}+1} y_{1}-\alpha y_{1}^{2} \\
& =\beta\left(p^{\nu_{1}+1}-y_{1}\right),
\end{aligned}
$$

and (7) reduces to $\alpha_{2} p^{\nu_{2}-\nu_{1}}=b_{\nu_{1}} \alpha_{1}-\beta_{1}$. Since $\operatorname{gcd}\left(\alpha_{1}, \beta_{1}\right)=1$, we must have $\operatorname{gcd}\left(\alpha_{2}, \alpha_{1}\right)=1$. By induction, we may assume in general that $\operatorname{gcd}\left(\alpha_{n+1}, \alpha_{n}\right)=1$.

(f) For $k_{1} \in\{1, \ldots, \alpha-\mu\}$, the numerator in (16) has the $\alpha$-part $<\alpha$, so we are done by induction on the $\alpha$-part. In addition, by the remark right after (17), we need only consider those $k_{1}$ for which, using also observation (a),

$$
k_{1}>K_{\alpha, \mu}:=\frac{(\alpha+1)(\mu-1)}{\mu} \geq(\mu-1)\left(1+\frac{1}{\mu}\right) .
$$

Returning to the proof, from (16), we have

$$
\begin{aligned}
A_{2} & =\frac{-\left(k_{1}+\mu-1\right) p^{2 \nu_{1}+1}}{\left(\mu+k_{1}-\alpha-1\right) p^{2 \nu_{1}+2}+\left(2 \alpha-\mu-k_{1}+1\right) p^{\nu_{1}+1} y_{1}-\alpha y_{1}^{2}} \\
& =: \frac{\alpha_{2} p^{\nu_{2}}}{\beta_{2}},
\end{aligned}
$$

where, if the step is not yet done by induction, by the above observations we may assume that

- $\alpha \geq \mu$

- the numerator $k_{1}+\mu-1$ has no extra power of $p$,

- there is no cancellation of the numerator and the denominator,

- $\operatorname{gcd}\left(\alpha, k_{1}+\mu-1\right)=1$,

- the denominator belongs to the range $\left((\mu-1) p^{2 \nu_{1}+2}, \mu p^{2 \nu_{1}+2}\right)$.

This implies in particular that $\nu_{2}=2 \nu_{1}+1$ and

$$
\begin{aligned}
\alpha<-\alpha_{2}= & k_{1}+\mu-1 \leq(\mu-1)+\alpha, \quad \operatorname{gcd}\left(\alpha, \alpha_{2}\right)=1, \\
(\mu-1) p^{\nu_{2}+1}<\beta_{2}= & \left(\mu+k_{1}-\alpha-1\right) p^{2 \nu_{1}+2} \\
& +\left(2 \alpha-\mu-k_{1}+1\right) p^{\nu_{1}+1} y_{1}-\alpha y_{1}^{2}<\mu p^{\nu_{2}+1} .
\end{aligned}
$$


Proceeding with the construction, we get

$$
\begin{aligned}
A_{2} & =\frac{-\left(k_{1}+\mu-1\right) p^{\nu_{2}}}{(\mu-1) p^{\nu_{2}+1}+x_{2}} \\
& =\frac{p^{\nu_{2}}}{p^{\nu_{2}+1}-y_{2}}-p^{\nu_{2}}\left(\frac{k_{1}+\mu-1}{(\mu-1) p^{\nu_{2}+1}+x_{2}}+\frac{1}{p^{\nu_{2}+1}-y_{2}}\right) \\
& =\frac{p^{\nu_{2}}}{p^{\nu_{2}+1}-y_{2}}-p^{\nu_{2}}\left\{\frac{\left\{k_{1}+2(\mu-1)\right\} p^{\nu_{2}+1}+x_{2}-\left(k_{1}+\mu-1\right) y_{2}}{(\mu-1) p^{2 \nu_{2}+2}+\left\{x_{2}-(\mu-1) y_{2}\right\} p^{\nu_{2}+1}-x_{2} y_{2}}\right\} \\
& =: \frac{p^{\nu_{2}}}{p^{\nu_{2}+1}-y_{2}}+A_{3} .
\end{aligned}
$$

Now, $p^{\nu_{2}+1} \mid\left\{x_{2}-\left(k_{1}+\mu-1\right) y_{2}\right\}$ and since $x_{2}, y_{2} \in D_{\nu_{2}}$, we have

$$
\begin{aligned}
x_{2}-\left(k_{1}+\mu-1\right) y_{2} & =:-\left(k_{1}-k_{2}\right) p^{\nu_{2}+1} \in\left\{-\left(k_{1}+\mu-2\right) p^{\nu_{2}+1}, \ldots,-p^{\nu_{2}+1}, 0\right\}, \\
k_{2} & \in\left\{-\mu+2, \ldots, k_{1}\right\}, \\
K_{k_{1}+\mu-1, \mu} & =\frac{\left(k_{1}+\mu\right)(\mu-1)}{\mu}>(\mu-1)\left(2-\frac{1}{\mu^{2}}\right) .
\end{aligned}
$$

The cases $1 \leq k_{1}+\mu-1 \leq K_{k_{1}+\mu-1, \mu}$ are handled by observation (f). Thus

$$
\begin{aligned}
A_{3} & =\frac{-\left\{k_{2}+2(\mu-1)\right\} p^{2 \nu_{2}+1}}{\left(\mu-1-k_{1}+k_{2}\right) p^{2 \nu_{2}+2}+\left(2 k_{1}-k_{2}\right) p^{\nu_{2}+1} y_{2}-\left(k_{1}+\mu-1\right) y_{2}^{2}} \\
& =: \frac{\alpha_{3} p^{\nu_{3}}}{\beta_{3}},
\end{aligned}
$$

where, if the step is not yet done by induction, by the observations we may assume, as before, that

- $\nu_{3}=2 \nu_{2}+1$,

- $\alpha<-\alpha_{3}=k_{2}+2(\mu-1) \leq 2(\mu-1)+\alpha$,

- $\operatorname{gcd}\left(\alpha_{2}, \alpha_{3}\right)=1$,

- $(\mu-1) p^{\nu_{3}+1}<\beta_{3}=\left(\mu-1-k_{1}+k_{2}\right) p^{2 \nu_{2}+2}+\left(2 k_{1}-k_{2}\right) p^{\nu_{2}+1} y_{2}$

$$
-\left(k_{1}+\mu-1\right) y_{2}^{2}<\mu p^{\nu_{3}+1} \text {. }
$$

Proceeding in the same manner and disregarding the cases where the conclusion can be settled by induction through the observations or the denominator falls inside the previous inductive range, in general, we consider

$$
\begin{aligned}
A_{n-1} & :=\frac{\alpha_{n-1} p^{\nu_{n-1}}}{\beta_{n-1}}=-\frac{k_{n-2}+(n-2)(\mu-1)}{\beta_{n-1}} p^{\nu_{n-1}} \\
& =-\frac{k_{n-2}+(n-2)(\mu-1)}{(\mu-1) p^{\nu_{n-1}+1}+x_{n-1}} p^{\nu_{n-1}}
\end{aligned}
$$




$$
\begin{aligned}
= & \frac{p^{\nu_{n-1}}}{p^{\nu_{n-1}+1}-y_{n-1}} \\
& -p^{\nu_{n-1}}\left\{\frac{k_{n-2}+(n-2)(\mu-1)}{(\mu-1) p^{\nu_{n-1}+1}+x_{n-1}}+\frac{1}{p^{\nu_{n-1}+1}-y_{n-1}}\right\} \\
= & \frac{p^{\nu_{n-1}}}{p^{\nu_{n-1}+1}-y_{n-1}} \\
& -\frac{\left\{k_{n-2}+(n-1)(\mu-1)\right\} p^{\nu_{n-1}+1}+x_{n-1}-\left\{k_{n-2}+(n-2)(\mu-1)\right\} y_{n-1}}{(\mu-1) p^{2 \nu_{n-1}+2}+\left\{x_{n-1}-(\mu-1) y_{n-1}\right\} p^{\nu_{n-1}+1}-x_{n-1} y_{n-1}} p^{\nu_{n-1}} \\
= & : \frac{p^{\nu_{n-1}}}{p^{\nu_{n-1}+1}-y_{n-1}}+A_{n},
\end{aligned}
$$

with $p \nmid x_{n-1} y_{n-1},(\mu-1) p^{\nu_{n-1}+1}<\beta_{n-1}<\mu p^{\nu_{n-1}+1}$ and

$$
0<x_{n-1}, y_{n-1}<p^{\nu_{n-1}+1}, \quad \alpha<-\alpha_{n-1} \leq(n-2)(\mu-1)+\alpha .
$$

By the algorithm, $p^{\nu_{n-1}+1} \mid\left\{x_{n-1}-\left(k_{n-2}+(n-2)(\mu-1)\right) y_{n-1}\right\}$ and since $x_{n-1}, y_{n-1} \in D_{\nu_{n-1}}$, we must have

$$
\begin{aligned}
& x_{n-1}-\left(k_{n-2}+(n-2)(\mu-1)\right) y_{n-1} \\
& \quad \in\left\{-\left(k_{n-2}+(n-2)(\mu-1)-1\right) p^{\nu_{n-1}+1}, \ldots,-p^{\nu_{n-1}+1}, 0\right\} .
\end{aligned}
$$

Here

$$
K_{k_{n-2}+(n-2)(\mu-1), \mu}=\frac{\left\{k_{n-2}+(n-2)(\mu-1)+1\right\}(\mu-1)}{\mu} \geq(n-1)(\mu-1) .
$$

Substituting

$$
x_{n-1}-\left\{k_{n-2}+(n-2)(\mu-1)\right\} y_{n-1}=-\left(k_{n-2}-k_{n-1}\right) p^{\nu_{n-1}+1}
$$

into (18), where, by observation (f), we need only consider

$$
\mu-1 \leq K_{k_{n-2}+(n-2)(\mu-1), \mu}-(n-2)(\mu-1)<k_{n-1} \leq k_{n-2} \leq \alpha,
$$

we get

$$
A_{n}=-\frac{k_{n-1}+(n-1)(\mu-1)}{\beta_{n}^{\prime}} p^{2 \nu_{n-1}+1}=: \frac{\alpha_{n} p^{\nu_{n}}}{\beta_{n}}
$$

where

$$
\begin{aligned}
\beta_{n}^{\prime}= & \left((\mu-1)+k_{n-1}-k_{n-2}\right) p^{2 \nu_{n-1}+2} \\
& +\left\{2 k_{n-2}+(n-3)(\mu-1)-k_{n-1}\right\} p^{\nu_{n-1}+1} y_{n-1} \\
& -\left\{k_{n-2}+(n-2)(\mu-1)\right\} y_{n-1}^{2} .
\end{aligned}
$$

As noted earlier, from the observations, we need only consider the possibility 
where

$$
\begin{aligned}
& \nu_{n}=2 \nu_{n-1}+1, \\
& \alpha<-\alpha_{n}=k_{n-1}+(n-1)(\mu-1) \leq(n-1)(\mu-1)+\alpha, \\
& \operatorname{gcd}\left(\alpha_{n-1}, \alpha_{n}\right)=1, \\
& (\mu-1) p^{2 \nu_{n-1}+2}<\beta_{n}^{\prime}=\beta_{n}=\beta_{n-1}\left(p^{\nu_{n-1}+1}-y_{n-1}\right)<\mu p^{2 \nu_{n-1}+2} .
\end{aligned}
$$

Going back to the old notation, we get

$$
\begin{aligned}
A_{n} & :=-\frac{k_{n-1}+(n-1)(\mu-1)}{\beta_{n}} p^{\nu_{n}} \\
& =\frac{p^{\nu_{n}}}{p^{\nu_{n}+1}-y_{n}}-p^{\nu_{n}}\left(\frac{k_{n-1}+(n-1)(\mu-1)}{\beta_{n}}+\frac{1}{p^{\nu_{n}+1}-y_{n}}\right) \\
& =\frac{p^{\nu_{n}}}{p^{\nu_{n}+1}-y_{n}}-p^{\nu_{n}} \frac{\left\{k_{n-1}+(n-1)(\mu-1)\right\}\left(p^{\nu_{n}+1}-y_{n}\right)+\beta_{n}}{\beta_{n}\left(p^{\nu_{n}+1}-y_{n}\right)} \\
& =\frac{p^{\nu_{n}}}{p^{\nu_{n}+1}-y_{n}}+p^{\nu_{n+1}} \frac{\alpha_{n+1}}{\beta_{n+1}}=: \frac{1}{a_{n}}+A_{n+1},
\end{aligned}
$$

where $a_{n}=b_{\nu_{n}} / p^{\nu_{n}}, b_{\nu_{n}}=p^{\nu_{n}+1}-y_{n}$. Again from the observations, we need only consider the possibility where, in the second term on the right-hand side of (20), the numerator has no extra power of $p$, there is no cancellation of the numerator and the denominator, the numerator is relatively prime to $\alpha_{n}$, and the denominator does not fall inside the previous inductive range. This leads, in particular, to $\nu_{n+1}=2 \nu_{n}+1$, and

$$
\beta_{n+1}=b_{\nu_{n}} \beta_{n}=\cdots=b_{\nu_{n}} \cdots b_{\nu_{1}} \beta_{1}=\left(p^{\nu_{1}+1}-y_{1}\right) \cdots\left(p^{\nu_{n}+1}-y_{n}\right) \beta_{1} .
$$

Thus, (7) reduces to

$$
\alpha_{n+1} p^{\nu_{n+1}-\nu_{n}}=b_{\nu_{n}} \alpha_{n}-\beta_{n} .
$$

Next, we establish some intermediate claims.

CLAIM 1. If there exists $c \in(0,1)$ such that $b_{\nu_{n}} / p^{\nu_{n}+1} \leq c$ for infinitely many $n$, then $\beta_{n}<(\mu-1) p^{\nu_{n}+1}$ for some sufficiently large $n$.

Proof. From (8) and (10), for $n$ sufficiently large we have

$$
\beta_{n+1}=b_{\nu_{n}} \cdots b_{\nu_{1}} \beta_{1}<c^{N} p^{\nu_{n}+\cdots+\nu_{1}+n} \beta<(\mu-1) p^{\nu_{n+1}+1},
$$

where $N$ is the number of indices $i \in\{1, \ldots, n\}$ for which $b_{\nu_{i}} / p^{\nu_{i}+1} \leq c<1$.

From Claim 1, we may further assume that for each $c \in(0,1)$, there exists $N_{c} \in \mathbb{N}$ such that $b_{\nu_{n}} / p^{\nu_{n}+1}>c$ whenever $n \geq N_{c}$; in particular, $b_{\nu_{n}} \rightarrow \infty$ as $n \rightarrow \infty$.

Claim 2. $\lim _{n \rightarrow \infty} p^{\nu_{n}}\left|\alpha_{n}\right| / \beta_{n}=\infty$. 
Proof. This follows immediately from the recurrence (7), i.e.,

$$
p^{\nu_{n+1}} \frac{\left|\alpha_{n+1}\right|}{\beta_{n+1}}=p^{\nu_{n}}\left(\frac{\left|\alpha_{n}\right|}{\beta_{n}}+\frac{1}{b_{\nu_{n}}}\right)>p^{\nu_{n}} \frac{\left|\alpha_{n}\right|}{\beta_{n}}+\frac{1}{p} .
$$

Claim 3. If there is a subsequence $\left\{n_{k}\right\} \subset \mathbb{N}$ such that the sequence of $\alpha$-parts $\left\{\left|\alpha_{n_{k}}\right|\right\}$ is bounded, then $\beta_{n}<(\mu-1) p^{\nu_{n}+1}$ for some sufficiently large $n$.

Proof. If the sequence $\left(\left|\alpha_{n_{k}}\right|\right)_{k}$ is bounded, then by Claim 2 the subsequence $\left(\beta_{n_{k}} / p^{\nu_{n_{k}}}\right)_{k}$ converges to 0 and the claim follows.

Claim 4. ||$\alpha_{n}|-| \alpha_{n-1}||<\alpha$ for $n \geq 1$.

Proof. Since

$$
\left|\alpha_{n}\right|=k_{n-1}+(n-1)(\mu-1) \quad \text { and } \quad\left|\alpha_{n-1}\right|=k_{n-2}+(n-2)(\mu-1),
$$

it follows that

$$
\left|\alpha_{n}\right|-\left|\alpha_{n-1}\right|=k_{n-1}-k_{n-2}+\mu-1 \leq \mu-1<\alpha
$$

and

$$
\left|\alpha_{n-1}\right|-\left|\alpha_{n}\right|=k_{n-2}-k_{n-1}-(\mu-1)<k_{n-2} \leq \alpha
$$

and the claim follows.

From Claim 3, we may further assume that the sequence of $\alpha$-parts, $\left(\left|\alpha_{n}\right|\right)_{n}$, has no bounded subsequences; in particular, it is never periodic. It follows that if the procedure does not end by induction after a sufficiently large number of operations, by (19), (20) and Claim 4, all $\alpha$-parts must be of the form $(\mu-1) m+i$ with large $m \in \mathbb{N}$ and $i \in\{1, \ldots, \alpha\}$. Let $P$ be the finite, possibly empty, set of all prime factors of $\mu-1$. If there are infinitely many $n$ such that all prime factors of $\beta_{n}$ belong to $P$, then since $\beta_{n+1}=\beta_{n} b_{\nu_{n}}$, it follows that the prime divisors of all $\beta_{n}$ and of all $b_{\nu_{n}}$ come only from $P$. Thus, there exists a prime $p_{0} \in P, p_{0} \neq p$, such that $p_{0} \mid \beta_{n}$ and $p_{0} \mid b_{\nu_{n}}$ and so $p_{0} \mid \beta_{n+1}$. But from (21), $p_{0} \mid \alpha_{n+1}$, which contradicts $\operatorname{gcd}\left(\alpha_{n+1}, \beta_{n+1}\right)=1$; this shows in particular that for the process to continue, we must have

$$
\operatorname{gcd}\left(b_{\nu_{n}}, \beta_{n}\right)=1 \text {. }
$$

Hence, there are infinitely many primes $q(\neq p)$ such that $q \mid \beta_{n}$ for all large $n$ but $q \nmid(\mu-1)$. Let $q_{0}, q_{1}, \ldots, q_{\alpha}$ be $\alpha+1$ such $q$ 's. By the Chinese remainder theorem, the system of $\alpha+1$ linear congruences

$$
\begin{aligned}
(\mu-1) m & \equiv 0\left(\bmod q_{0}\right), \\
(\mu-1) m & \equiv-1\left(\bmod q_{1}\right), \\
& \vdots \\
(\mu-1) m & \equiv-\alpha\left(\bmod q_{\alpha}\right),
\end{aligned}
$$


has a solution $m_{0}$ sufficiently large, i.e.,

$$
q_{i} \mid\left\{(\mu-1) m_{0}+i\right\} \quad(i=0,1, \ldots, \alpha) .
$$

This implies that for $n$ sufficiently large, there is a cancellation of the numerator and the denominator of $A_{n+1}$, and we are done by observation (c). This finishes the proof of Lemma 6 and completes the proof of the theorem.

\section{References}

[1] P. J. Grabner and A. Knopfmacher, Arithmetic and metric properties of p-adic Engel series expansions, Publ. Math. Debrecen 63 (2003), 363-377.

[2] A. Knopfmacher and J. Knopfmacher, Series expansions in p-adic and other nonarchimedean fields, J. Number Theory 32 (1989), 297-306.

[3] —, - Infinite series expansions for p-adic numbers, ibid. 41 (1992), 131-145.

[4] V. Laohakosol and N. Rompurk, A characterization of rational elements by Lürothtype series expansions in the p-adic number field and in the field of Laurent series over a finite field, Acta Arith. 122 (2006), 195-205.

[5] V. Laohakosol, N. Rompurk and A. Harnchoowong, Characterizing rational elements using Knopfmachers' expansions in the field of Laurent series over a finite field, Thai J. Math. 4 (2006), 223-244.

Department of Mathematics

Kasetsart University

Bangkok 10900, Thailand

E-mail: fscivil@ku.ac.th
Department of Mathematics

Khon Kaen University

Khon Kaen 40002, Thailand

E-mail: rompurk@hotmail.com

Received on 30.5.2007

and in revised form on 2.11.2007 